\title{
FIXED-BED ADSORPTION SEPARATION OF XYLENE ISOMERS OVER SIO2/SILICALLITE-1 CORE-SHELL ADSORBENTS
}

\author{
Easir A. Khan ${ }^{1 *}$, Arvind Rajendran ${ }^{2}$, Zhiping Lai ${ }^{3}$ \\ ${ }^{1}$ Department of Chemical Engineering, Bangladesh University of Engineering \& Technology, Dhaka-1000, \\ Bangladesh \\ ${ }^{2}$ Department of Chemical \& Material Science, University of Alberta, Canada \\ ${ }^{3}$ Division of Physical Sciences and Engineering, King Abdullah University of Science and Technology, Thuwal \\ 23955-6900, Kingdom of Saudi Arabia
}

Received 4 May 2013; received in revised form 17 September 2013

\begin{abstract}
SiO}_{2} /$ Silicalite-1 core-shell material has been demonstrated as potential shape selective adsorbent in gas phase separation of $\mathrm{p}$-xylene from a mixture of p/o-xylene isomers. The core-shell composite comprised of large silica core and thin polycrystalline silicalite-1 shell which was synthesized via a self-assembly of silicalite-1 nanocrystals on core silica surface followed by a secondary seeded growth method. The core materials, $\mathrm{SiO}_{2}$ used in this study has mesoporosity with an average pore diameter of $60 \AA$ and hence offers no shape selectivity for xylene isomers. However, the shell, silicalite-1 contains rigid pore structures and preferentially adsorbs p-xylene from their isomers mixtures. A series of adsorption fixed bed breakthrough adsorption/desorption experiment was performed to obtain the equilibrium isotherms and adsorption isotherm parameters of xylene isomers. The equilibrium isotherms of xylene isomers follow the Langmuir's model. A chromatographic adsorption model has been used to describe the fixed-bed breakthrough profiles of xylene isomers. The model has successfully predicted the responses of the binary mixtures of p/o-xylene isomers. The $\mathrm{SiO}_{2} /$ silicalite- 1 core-shell adsorbents have shown para-selectivity as high as 15 .
\end{abstract}

Keywords: Kinetic Separation; Xylene Isomers; Core-Shell; Zeolite; Adsorption.

\section{INTRODUCTION}

Adsorption based separation process are widely used in the industry for separation of several close boiling point hydrocarbon mixtures during the past three decades [1-3]. Zeolite molecular sieves have been employed as a major adsorbent material in many industrial separation processes for separation of various organic mixtures. Synthetic zeolite crystals are often a few microns in size that introduces pressure drop problems if used in the original form. The pressure drop increases the overall operational cost in adsorption process. Very often, the crystals are formed into a macroporous pellet of suitable dimension, size and porosity to make it a commercially viable adsorbent. Several discoveries and international patents have been reported for the development of efficient methodologies of pellet formation and preparation of commercials adsorbents [4]. However, the pelletalization of these materials can introduce additional mass transfer resistances leading to longer cycle times and 
hence high operational costs. Thus the development of novel adsorbents with higher mass transport rates has potential in the adsorption based separation processes for both in chemical and petrochemical industry.

Zeolites, as adsorbents, have been extensively used in the hydrocarbon separation in petroleum or petrochemical industries [2, 5-10]. Zeolite core-shell composites are novel class of materials for their potential application in the field of catalysis, adsorption, and storage and controlled release [11-15]. The application of these materials in adsorption based separation process has not been fully explored. Very few reports have been found in the literatures. For instance, Bouizi et al. studied the adsorption of aromatic hydrocarbon using $\beta$-zeolite/silicalite-1. This core-shell adsorbent was composed of $15 \mu \mathrm{m} \quad \beta$-zeolite core and 3 layer of silicalite- 1 shell (thickness $\sim 1.5 \mu \mathrm{m}$ in first layer). The crystallization of silicalite-1 shell was initiated by electrostatically adsorbed silicalite- 1 nanoseeds and covered $86 \%$ of $\beta$ zeolite crystals during the secondary growth. Bouizi et al. investigated the adsorption properties of butane, toluene and 1,3,5trimethylbezene using core $\beta$-zeolite, silicalite-1 and $\beta$-zeolite/silicalite-1 coreshell composite. The pore of $\beta$-zeolite is large enough for adsorbing these molecules while shell silicalite-1 limits the access of trimethylbenze. This is because of that the pore size of silicalite- 1 is smaller than the kinetic diameter of trimethylbezene. They have shown that silicalite-1 particles $(\sim 1 \mu \mathrm{m})$ have less capacity of adsorption of trimethylbezene in comparison with $\beta$ zeolite core and the capacity of adsorption was even much lower when core-shell particles used as adsorbent [15]. Casado et al. reported the gas adsorption properties of hollow zeolite sphere [13]. They synthesized hollow silicalite-1 shell using mesoporous silica sphere $(\sim 4 \mu \mathrm{m})$ as core where the core silica was converted into hollow shell during the hydrothermal synthesis. The hollow silicalite-1 shell showed the same adsorption capacity as silicalite-1 particles for small hydrocarbon molecules like propane. However, the materials showed high capacity for the adsorption of 1,3,5-triisopropylbezene. While such hollow adsorbent materials are useful for storage and control release of large molecules, this indicates the presence of non-zeolitic pores or defects in the shell membrane as the large molecule like 1,3,5-tri-isopropylbezene can only penetrate through the defects of the shell.

The work focuses on the development of core-shell adsorbents with a mesoporous interior and microporous thin defect-free shell and their application in the adsorption based separation process of xylene isomers, more specifically $p$-xylene and o-xylene. The separation of the xylene isomers in pure form is an important and challenging problem in the chemical and petrochemical industries because of its close boiling points and high market for one of the isomers. The industrial separation processes of xylene isomers are often coupled with an isomerization process. The end products of the isomerization process are ethylbezene, oxylene, m-xylene and p-xylene. The integration of separation process with isomerization is intended to transform all the other forms to p-xylene. Because of the fact that $p$-xylene has increased industrial importance, especially for the large scale manufacturing of terapthalic acid which is the raw materials for the production of polyester or plastic.

A number of methods can be applied for the separation of xylene isomers, e.g. extract distillation, fractional crystallization or selective adsorption [16]. Currently, most adsorption processes are based on selective 
adsorption on molecular sieve materials because of process simplification, less energy requirement and enhancement of productivity. The adsorption units of Parex process developed by UOP have been commercialized for the separation of $\mathrm{p}$ xylene from its mixture. The adsorbent used in the process is FAU type of zeolites, especially potassium-exchanged zeoliteY [16-18]. Most processes involving separation of xylene isomers are performed in the liquid phase. Such processes have been investigated thoroughly both experimentally and theoretically by Santacesaria et al [16, 18, 19]. Faujasite zeolites have been extensively studied as adsorbent for the separation of $\mathrm{p} / \mathrm{m}$-xylene in vapor phase [20-23]. It is well known that the fuajasite zeolite can separate xylene isomers from it mixtures. The pore size of faujasite zeolite is larger than the kinetic diameter of xylene isomers. The sieving effect of faujasite zeolite is mainly dependent on the pore filling, fluid phase concentration and exchangeable cation [20]. Recently, US Patent by Doyle et al. disclosed the efficiency of xylene isomer separation from a mixture utilizing MFI zeolite comprising of zeolite crystals and a binder [4]. Dessau et al. reported that the medium pore ZSM-5 zeolite can preferentially adsorb p-xylene from p/oxylene mixture with a para-selectivity of 5 based on liquid phase adsorption using mesityline as solvent [24]. The adsorption of liquid phase separation system is usually low temperature, however, the range of temperature for industrial gas phase separation system is $150^{\circ} \mathrm{C}-175^{\circ} \mathrm{C}$ [25]. Though, the liquid phase separation process is more energy consuming, the process perhaps becomes less suitable in comparison with the gas phase process in terms of process efficiency. The selectivity in gas phase adsorption separation process is comparatively low; however, the process is much more beneficial because of the following reasons, i.e. a) low mass transfer resistance, b) negligible axial dispersion and c) much lower hold up in the adsorbent bed. Several reports have been published with a detailed comparison of gas phase and liquid phase separation process [25-28]. After a comprehensive study of gas phase and liquid phase processes, Morbidelli et al. concluded that gas process is much more efficient than liquid phase adsorption considering all the process parameter such as mass transport rate, hold up, capacity of adsorption, axial dispersion, and energy consumption [25].

In this work, we have studied the separation performance of p-,o-xylene isomers by fixed-bed adsorption breakthrough experiment over $\mathrm{SiO}_{2} /$ Silicalite- 1 core-shell adsorbents. The core-shell composite was synthesized by a seeded growth technique reported in literatures [12,29]. The core materials used in this study is mesoporous silica with an average pore diameter of $60 \AA$ which is several order of magnitude larger than the molecular dimension of xylene isomers. Hence, the core has ideally no shape selectivity for xylene isomers. Besides, the shell, silicalite-1, contains rigid pore structures and is capable of adsorbing molecules on the basis of their kinetic diameter of the molecules from a mixture. The layer of silicalite-1 is a well proven membrane material and possess high resolution shape selectivity for the sieving the p-xylene from its isomer mixtures. The silicalite-1 layer on a macroporous support has been extensively studied over the decades for not only in the separation of various organic isomers but also for the application in chemical sensor and catalysis [30-35].

The core-shell adsorbents composed of a thin shell possess several advantages over the conventional pelleted adsorbent 
materials: First, the effective adsorption occurred in the thin zeolite layer. It has no macroporosity that produces extra mass transfer resistance in case of pelleted adsorbents. Second, both the particle size and shell thickness of the core-shell adsorbents can be tuned. The silica core with various size and shape is commercially available and the shell thickness can be controlled from $40 \mu \mathrm{m}$ to $1 \mu \mathrm{m}$ by adjusting the synthesis parameters of secondary growth $[12,36]$. Moreover, minimizing of shell thickness or effective adsorption length, the periodic/cycling time of adsorption process can be reduced to a great extent which has an overall impact on the process economy. Hence, the core-shell composite adsorbent materials can be envisioned as possible alternatives to address the current bottlenecks of the existing adsorption process.

\section{EXPERIMENTAL}

\section{Synthesis of Core-Shell Adsorbents}

A seeded growth method discussed in details in our previous report [12] was used to synthesize $\mathrm{SiO}_{2} /$ silicallite-1 core-shell particles. It includes three steps: First, the core surface was modified by layer of polyelectrolytes. Second, the modified core surface was coated with a monolayer of zeolite nanocrystals via self-assembly process. Finally, the seed layer was converted into a compact and well intergrown shell via secondary growth. The zeolite seed nanocrystals $(\sim 500 \mathrm{~nm})$ were synthesized following the recipe of $5 \mathrm{SiO}_{2}: 1$ TPAOH: 16 EtOH: $250 \mathrm{H}_{2} \mathrm{O}$.

The surface of core particles was modified by repeated self-assembly of two strong polyelectrolytes (PE) such as polydiallyldimethylammonium chloride (PDDA, $\quad M_{\mathrm{w}} \sim 100,000-200,000, \quad 20 \%$ in water, Aldrich); and sodium polystyrenesulfonate (PSS, $M_{\mathrm{w}} \sim 70,000$, Aldrich). The details of self-assembly process can be found in literatures [12, 29]. In a typical coating process, $0.5 \mathrm{~g}$ of $\mathrm{SiO}_{2}$ particles was immersed into $40 \mathrm{ml}$ of a polyelectrolyte solution $\left(\sim 1 \%\right.$ in $\left.\mathrm{H}_{2} \mathrm{O}\right)$ and the surface properties to electro-positive by layer-by-layer self-assembly of polyelectrolyte following the sequence of $\mathrm{SiO}_{2} / \mathrm{PDDA} / \mathrm{PSS} / \mathrm{PDDA}$. Next, the core particles were immersed into $10 \mathrm{ml}$ of seed suspension to form a seed monolayer on the surface.

The synthesis solution of secondary growth was prepared by adding $4 \mathrm{~g}$ of TPAOH aqueous solution $\left(\sim 40 \%\right.$ in $\left.\mathrm{H}_{2} \mathrm{O}\right)$ and $8 \mathrm{~g}$ of TEOS $(298 \%)$ in $144 \mathrm{~g}$ of deionized water. The mixture was stirred at room temperature for about $4 \mathrm{~h}$ and mixed with $1 \mathrm{~g}$ of seed-coated $\mathrm{SiO}_{2}$ and the resulting mixture was then transferred to a Teflon-lined autoclave. The autoclave was placed in a preheated oven and heated at temperature $448 \mathrm{~K}$ for a duration of $24 \mathrm{~h}$ under rotation. After being cooled, the resulting particles were separated by filtration and washed with deionized water. The core-shell particles were finally calcined at $753 \mathrm{~K}$ for $10 \mathrm{~h}$ with ramping rate of $0.5 \mathrm{~K}$ under air flow.

\section{Experimental Setup for Dynamic Column Breakthrough}

The gas phase adsorption of organic mixtures was performed in an adsorption unit which is schematically shown in Figure 1. The adsorption column was $77 \mathrm{~mm}$ in length and $4.46 \mathrm{~mm}$ in diameter. The packed length of the column was $67.2 \mathrm{~mm}$ while the rest was filled with glass-wool. The column packing was accomplished by slurry packing. The adsorbents was mixed with methanol to form a slurry and pumped into the column. The column was then evacuated at $473 \mathrm{~K}$ for $5 \mathrm{~h}$ to remove the adsorbed 
methanol. Afterwards, the column was placed in a heater and slowly heated to $423 \mathrm{~K}$

All Mass Flow Controllers (MFC) were connected to a $\mathrm{N}_{2}$ cylinder. Moisture in the gas was removed using a guard bed. MFC-1 was activated with a constant flow rate of 10 $\mathrm{mL} / \mathrm{min}$ through the column. A bubbler was placed downstream of MFC-2 at room temperature, which allowed the saturation of the gas that flowed through it. The flow of MFC-3 was regulated in oder to suitably dilute the gas stream. The total flow of MFC-2 and MFC-3 was maintained at 10 $\mathrm{mL} / \mathrm{min}$.

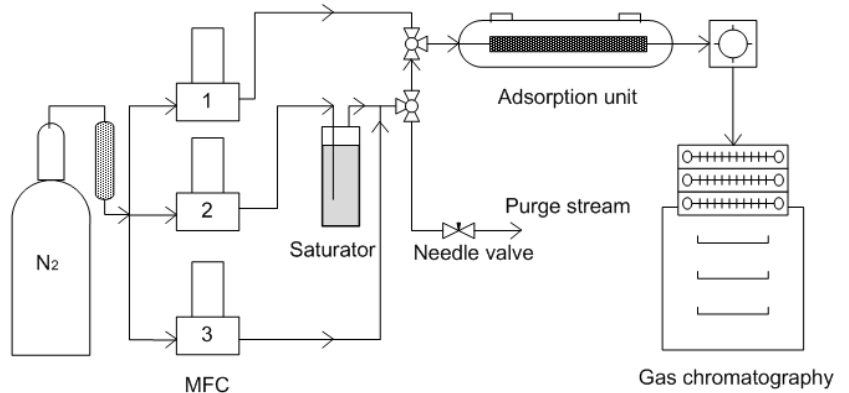

Figure 1: Schematic Representation of Continuous Flow Fixed Bed Adsorption/Desorption System, (MFC - Mass Flow Controller)

Initially the column was purged using pure $\mathrm{N}_{2}$ using MFC1. During this time, MFC2 and MFC3 were activated and the mixture is purged. At time $\mathrm{t}=0$, using the three way valve, the feed was switched from MFC 1 to the feed stream. Simultaneously, the gas chromatography unit (Agilent 7890 with a FID detector and a Zebron ZB-WAX plus column) was started which collects a sample every 3 minutes. The sample was automatically injected into the GC. The flow was continued for 60 mins, after which the column was desorbed using $\mathrm{N}_{2}$. During the entire period the flow rate was measured downstream of the column using a bubble flow meter.

\section{RESULTS AND DISCUSSION}

Figure $2 \mathrm{a}$ shows the scanning electron microscopy images of core-shell particles. The top surface of a core-shell particle can be seen from higher magnified image that shown in Figure 2b. All the core-shell particles are composed of a continuous layer of silicalite- 1 and no visible cracks or defects are observed under SEM. A preferentially c-oriented well-intergrown shell is formed on the outer surface of core particles during the secondary growth. The average shell thickness is $1.5 \mu \mathrm{m}$ that can be measured from SEM image of broken coreshell particles. An image of broken coreshell particle is shown in Figure 2c.
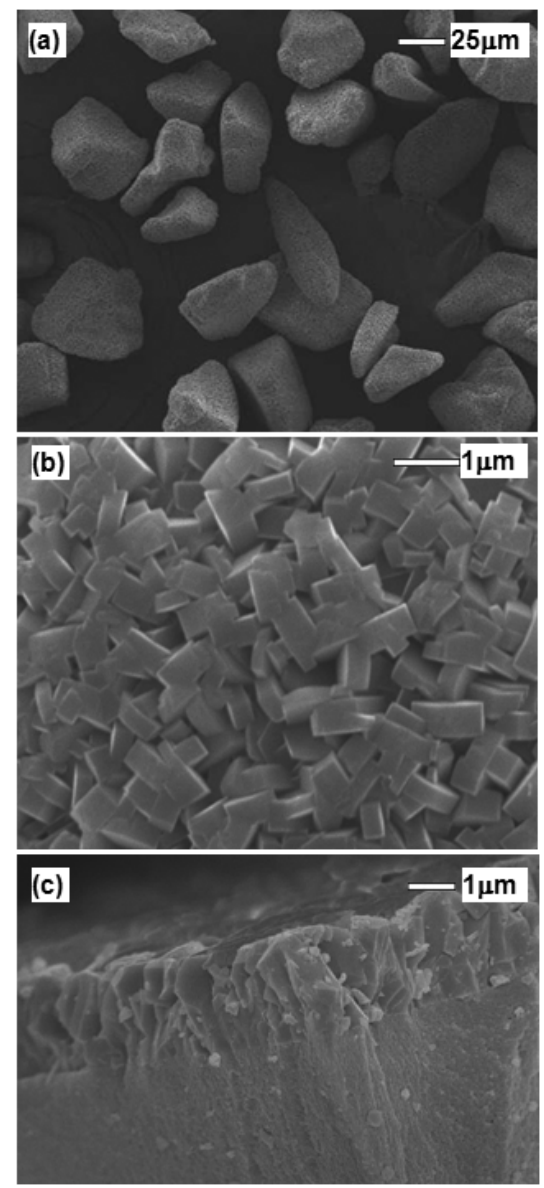

Figure 2: SEM Images of Core-Shell Particles, Top and Cross-Sectional View of Core-Shell Adsorbents 
Table 1: Chemical Composition and Textural Characteristics of the Supports $\mathrm{SiO}_{2}$, and $\mathrm{SiO}_{2} /$ Silicallite-1 Core-Shell

\begin{tabular}{cccc}
\hline Adsorbent & $\begin{array}{l}\text { BET surface } \\
\text { area }\left(\mathrm{m}^{2} / \mathrm{g}\right)\end{array}$ & $\begin{array}{l}\text { Average pore } \\
\text { diameter }(\mathrm{nm})\end{array}$ & $\begin{array}{l}\text { Pore volume } \\
\left(\mathrm{cm}^{3} / \mathrm{g}\right)\end{array}$ \\
\hline $\mathrm{SiO}_{2}$ & 456 & 62 & 0.83 \\
$\mathrm{SiO}_{2} /$ Silicallite-1 & 238 & 43 & 0.21
\end{tabular}

\section{Physical and Chemical Properties of Core and Core-Shell Particles}

The specific surface area, pore size distribution and pore volume of core and core-shell particle was measured by $\mathrm{N}_{2}$ sorption at $77 \mathrm{~K}$ using Quantachrome autosorb gas adsorption system and their values are tabulated in Table 1 . One can see that the BET surface and average pore size of core silica is higher and decreased during the formation of core-shell particles. The pore size distribution of core $\mathrm{SiO}_{2}$, and coreshell $\mathrm{SiO}_{2} /$ silicalite- 1 were estimated from the desorption curve of $\mathrm{N}_{2}$ sorption isotherm using $\mathrm{BJH}$ methods. The pore sizes of distributions of different materials are shown in Figure 3 (inset).

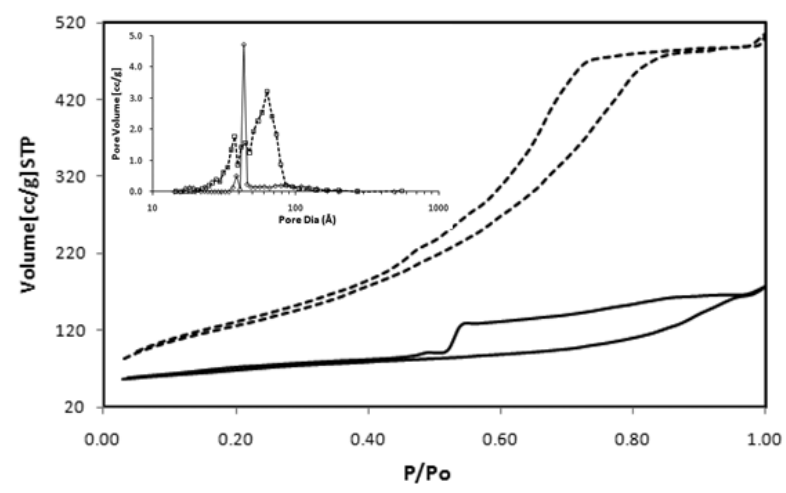

Figure 3: $\mathrm{N}_{2}$ Sorption Isotherms (at $77 \mathrm{~K}$ ) of $\mathrm{SiO}_{2}$ and $\mathrm{SiO}_{2} /$ silicalite-1, and Inset Shows the Pore Size Distributions

The core materials $\mathrm{SiO}_{2}$ have pattern of dispersed pore size distribution and a maximum of distribution curve at $62.99 \AA$. However, it is interesting that the pore size distribution becomes narrower for the coreshell particles with an average value of 43 $\AA$. It may be cause of that the structural change of core materials either in secondary growth step or high temperature calcination step. The exact reason behind this is surprising and has not been investigated yet. The core-shell particle is a hierarchical structure; the shell is microporous and the core is mesoporous. It can be expected that there might be gaps between the core and the shell with an unknown size distribution. Hence a single nitrogen adsorption experiment is expected to yield a composite measurement of all these and can overshadow the information from the shell alone. The hysteresis observed in Figure 3 from sorption isotherm of $\mathrm{SiO}_{2}$, suggested that the materials contain mesopore of properly defined cylindrical like pore or agglomerates of compact of roughly homogeneous spheres. The type of hysteresis loop observed for core-shell materials is associated with thin slit pores in mesopore region and also including pores in micropore region. This clearly indicates the fact of the mixing effects of mesopore of core and micropore of shell. However, it is indeed interesting that the pore size distribution becomes narrower and average size is decreased. Adsorpiton of xylenes on bare core $\mathrm{SiO}_{2}$ particles

The core silica particle is mesopous and has extra-large pore size in compare to molecular dimension. So it has ideally no size exclution effects. The breakthrough adsorption experiment was performed to investigate the separation effects of 50/50 mixture of p-xylene and o-xylene. The adsorption behaviours observed are similar from their breakthrough profiles for both $\mathrm{p}$ xylene and o-xylene that shown in Figure 4. This indicates that the core particles have no shape selectivity. However the behaviours observed in core-shell particles are completely different that has been discussed 
in details in the following sections.

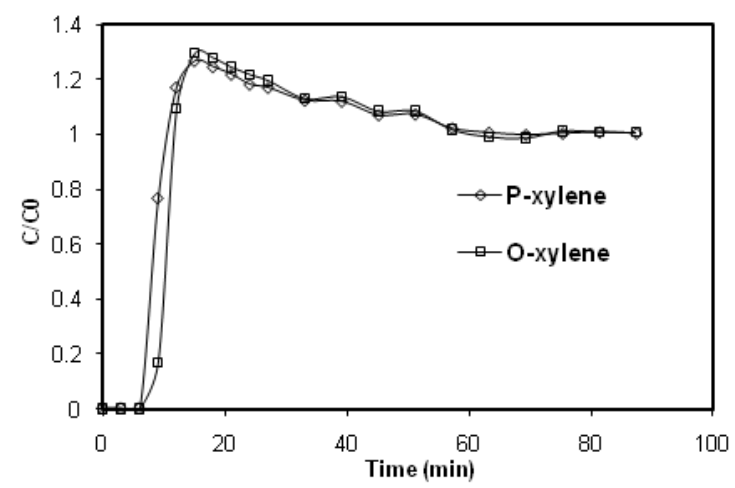

Figure 4: Breakthrough Profiles of p/oXylenes for Core Silica particles with a constant flow of $10 \mathrm{~mL} / \mathrm{min}$ [STP] at $150{ }^{\circ} \mathrm{C}$

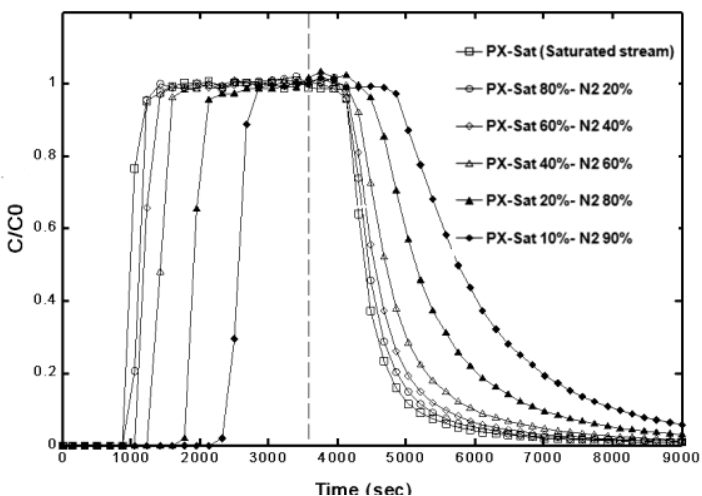

Figure 5: Single Component Breakthrough curves of p-Xylene at Different Inlet Concentrations with a Constant Flow of 10 $\mathrm{mL} / \mathrm{min}[\mathrm{STP}]$ at $423 \mathrm{~K}$

\section{Single Component Breakthrough Experiments of p-Xylene and o-Xylene}

The isotherm of an adsorbent material is an essential characteristic that provides the capacity of adsorption of a material as a function of fluid phase concentration. It helps to explain the adsorption phenomenon, their nature and physical significances. The adsorption isotherm of core-shell adsorbents for a component can be obtained from the single component fixed bed adsorption breakthrough curves at isothermal condition. The breakthrough experiments were performed at $423 \mathrm{~K}$ in this study. The temperature range for industrial gas phase separation process of xylene isomer is 423$453 \mathrm{~K}$.

A series of experiments for both p/oxylene isomers were conducted with different fluid phase concentrations. The normalized concentration (with respect to inlet concentrations) profiles of $\mathrm{p}$-xylene are shown in Figure 5. The feed concentrations were adjusted by mixing of two steams; one is saturated $\mathrm{N}_{2}$ gas stream that bubbles through liquid p-xylene or o-xylene and other is pure $\mathrm{N}_{2}$ gas stream. The outlets of adsorption unit were continuously injected by an autosampler in a gas chromatograph and analyzed in each three minutes of interval. The data was plotted as normalized concentration versus time to construct the breakthrough curves. The nature of the isotherm can be comprehended from the dynamic response of breakthrough experiments. The responses of $\mathrm{p}$-xylene can be seen from Figure 5. The mean retention times of breakthrough experiments are dependent on the feed concentration. In this case, it decreases with the increase of feed concentration. It has a sharp adsorption front and diffuse desorption that can be seen for all the breakthrough curves. Both characteristics indicate that the equilibrium isotherm is favorable for $\mathrm{p}$-xylene.

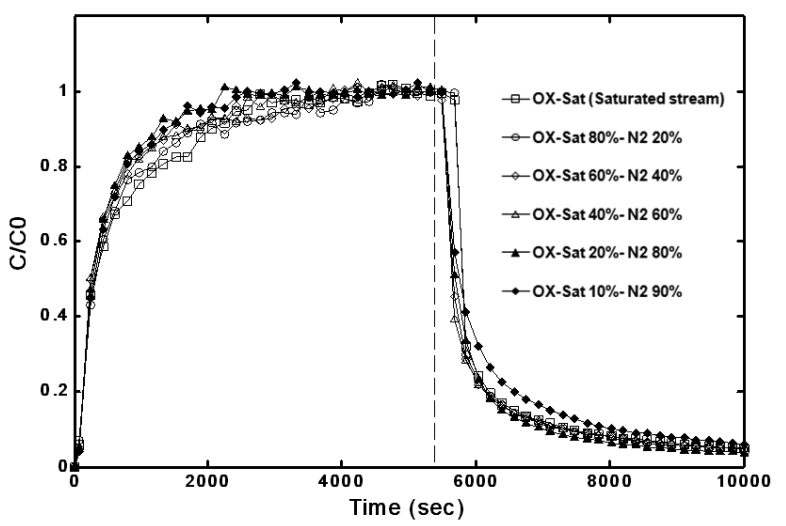

Figure 6: Single Component Breakthrough Curves of o-Xylene at Different Feed Concentrations with a Constant Flow of 2 $\mathrm{mL} / \mathrm{min}[\mathrm{STP}]$ at $423 \mathrm{~K}$ 
The breakthrough profiles for o-xylene are shown in Figure 6. It can be observed that for all concentrations explored, the profiles are nearly identical. The concentration front shows a constant pattern that indicates a linear equilibrium isotherm. However, compared to p-xylene the effect of mass transfer resistance is more evident as seen from the long tails for the adsorption and desorption fronts.

\section{Equilibrium Adsorption Isotherms, Henry's Constant and Shape Selectivity of Core-shell Adsorbents for Separation of p/o-Xylene Isomers}

The representation of an adsorption process in fixed-bed column loaded with solid sorbent materials is shown in Figure 7. The bed has a length $L$, and a void fraction $\varepsilon$ and fluid flows through the bed with superficial velocity $v$.

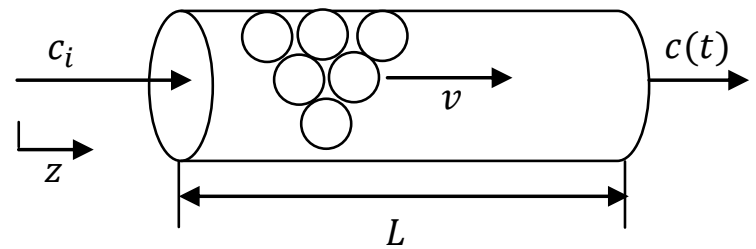

Figure 7: Scheme of a Packed Bed Column Loaded with Adsorbents

For an adsorption breakthrough a simple mass balance across the column, $(\mathrm{In}-\mathrm{Out}=$ Accumulation), can be written as,

$$
\begin{gathered}
v \in A c_{i} \int d t-v \in A \int c(t) d t=\epsilon c_{i} V_{b e d} \\
+(1-\epsilon) V_{b e d} q^{*}
\end{gathered}
$$

The expression for breakthrough time, $[\mathrm{c}(\mathrm{t})=0]$, yields from Eqn 5.1

$$
\begin{aligned}
t_{\text {breakthrough }} & =\int\left(1-\frac{c(t)}{c_{i}}\right) d t \\
& =\frac{L}{v}\left[1+\frac{(1-\epsilon)}{\epsilon} \frac{q^{*}}{c_{i}}\right]
\end{aligned}
$$

The single component equilibrium adsorption capacity or loading was estimated from the breakthrough experimental data. The equilibrium adsorption capacity of each concentration is calculated by such a procedure as in Eqn 2 . We observed that the adsorption capacity of $\mathrm{p}$-xylene is significantly higher than that of $\mathrm{o}$-xylene. This is because of the fact that $\mathrm{p}$ xylene is easily accessible to the porous networks of the core-shell adsorbents. On the other hand, o-xylene is unable to penetrate through the pore of the adsorbent's shell because of having larger kinetic diameter.

It is worth noting that the mean retention time obtained from the normalized concentration profiles is corrected by subtracting the retention time caused by the dead volume. The breakthrough experiment for dead volume was conducted by replacing the column with a union connection. Based on the equilibrium data and breakthrough behavior; we find that the isotherm is favorable for p-xylene and non-linear. Hence, a Langmuir isotherm can be used for fitting the isotherm data to find the sorption properties of the core-shell adsorbents. The single site Langmuir model equation can be expressed as,

$$
q_{i}^{*}=q_{s a t} \frac{b c}{1+b c}
$$

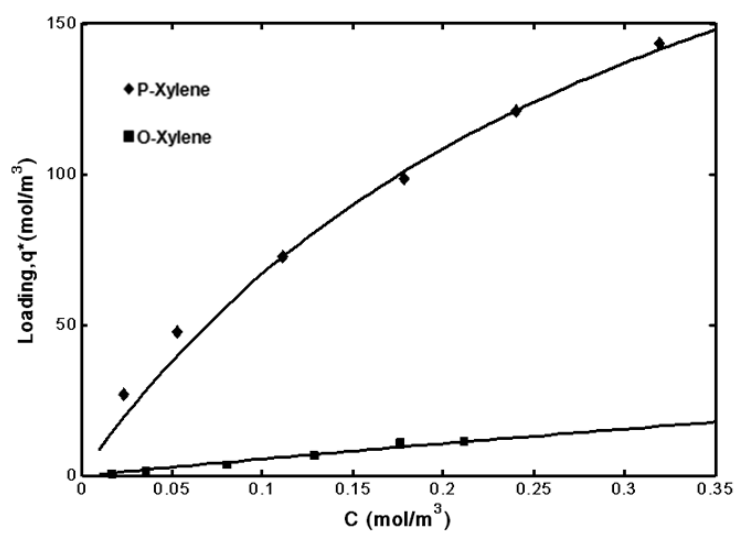

Figure 8: Adsorption Isotherms of Xylene Isomers, Symbols are Experimental Data and Solid Lines are Fitted Data by Langmuir 8 Model 
Where $q_{\text {sat }}$ is the saturation loading of adsorbent and $b$ is the adsorption equilibrium constant. Figure 8 shows the adsorption isotherm of p-xylene and oxylene.

The solid lines of Figure 8 represent the fitted data of Langmuir model of Eqn 3. The adsorption isotherm of o-xylene is observed linear while $p$-xylene is non-linear. The similar nature of isotherms has been suggested from their response of breakthrough experiments in previous section. The Langmuir parameters $q_{\text {sat }}$ and $b$ are estimated from the data fitting in Eqn 3. The Henry's law constant can be obtained from the Langmuir parameters, as $\mathcal{H}=$ $q_{s a t} b$ since the experiments were conducted at low fluid phase concentration. The Langmuir sorption parameters and Henry's law constant data of core-shell adsorbents are given in Table 2 .

Table 2: Sorption Parameters of Core-Shell Adsorbents at $150{ }^{\circ} \mathrm{C}$

\begin{tabular}{ccccc}
\hline Component & $\begin{array}{c}\text { Saturation } \\
\text { Loading, } q_{\boldsymbol{s}} \\
\left(\mathbf{m o l}_{\mathbf{m}} \mathbf{m}^{\mathbf{3}}\right)\end{array}$ & $\begin{array}{c}\text { Equilibrium } \\
\text { Constant, } \boldsymbol{b}, \\
\left(\mathbf{m}^{\mathbf{3} / \mathbf{m o l})}\right.\end{array}$ & $\begin{array}{c}\text { Henry Constant, } \\
\boldsymbol{H}=\boldsymbol{b} \boldsymbol{q}_{\boldsymbol{s}}\end{array}$ & $\begin{array}{c}\text { Selectivity } \\
\boldsymbol{\alpha}_{\mathbf{p} / \text {-Xylene }}\end{array}$ \\
\hline P-xylene & 286.78 & 3.05 & 872.09 & 15.2 \\
O-xylene & 154.76 & 0.3702 & 57.29 & \\
\hline
\end{tabular}

Table 3: Comparison of Shape Selectivity of Different Adsorbents for the Separation of $\mathrm{p}$ Xylene from $\mathrm{p} / \mathrm{o}-$ Mixture $\left({ }^{\mathrm{b}} \mathrm{p} / \mathrm{m}\right.$-mixture $)$

\begin{tabular}{|c|c|c|c|c|}
\hline Material & Temperature $\left({ }^{\circ} \mathrm{C}\right)$ & $\begin{array}{l}\text { Adsorption } \\
\text { Process }\end{array}$ & Para-Selectivity & Reference \\
\hline ZSM-5 & 25 & Liquid phase & $3-16$ & Yan et a1. 13,38 \\
\hline MILL-53 & 25 & Liquid phase & 3.5 & Alaerts et al..$^{39}$ \\
\hline Faujasite & $\begin{array}{l}25 \\
170\end{array}$ & $\begin{array}{l}\text { Liquid phase } \\
\text { Gas phase }\end{array}$ & $\begin{array}{l}\leq 3 \\
\leq 1.5\end{array}$ & Ruthven et al. 40 \\
\hline Zeolite Y & $\begin{array}{c}20 \\
150\end{array}$ & $\begin{array}{l}\text { Liquid phase } \\
\text { Gas phase }\end{array}$ & $\begin{array}{c}\sim 4.5-5.5 \\
\leq 3\end{array}$ & $\begin{array}{c}\text { Santacesaria et } \\
\text { al. } \text {. }^{19,27}\end{array}$ \\
\hline $\mathrm{BaY}$ or $\mathrm{NaY}$ & 150 & Gas phase & $\leq 2.5^{\mathrm{b}}$ & Cottier et al..$^{20}$ \\
\hline $\mathrm{SiO}_{2} /$ Silicalite-1 & 150 & Gas phase & 15.24 & This work \\
\hline
\end{tabular}

The selectivity for core shell particles is 15.24 which calculated from the ratio of the Henry's constant as shown in Table 2. Adsorption separation selectivity of p/o-xylene isomer for MFI zeolite is about 5 or more that has been reported in the literatures [24]. The comparison of selectivity for separation of $\mathrm{p}$-xylene from their isomer for different adsorbent materials in gas or liquid phase process is shown in Table 3. The gas phase separation selectivity of xylene isomers is less than 3 [25, 28]. Thus, the gas phase separation selectivity has enhanced significantly for core-shell type of adsorbent used in this study.

Table 4: Comparison of Adsorption Capacity and Selectivity of Core, Shell Particles and Core-Shell Materials for Binary Adsorption at $150^{\circ} \mathrm{C}$

\begin{tabular}{ccc}
\hline Adsorbents & p-Xylene $(\mathbf{m g} / \mathbf{g})$ & o-Xylene $(\mathbf{m g} / \mathbf{g})$ \\
\hline $\mathrm{SiO}_{2}$ & 1.62 & 1.59 \\
Silicalite-1 & 8.4 & 3.58 \\
$\mathrm{SiO}_{2} /$ Silicalite-1 & 3.97 & 0.25 \\
\hline
\end{tabular}

A comparative study was performed to investigate the adsorption capacity of core particles, shell particles and core-shell particles as shown in Table 4. The core particles show no preference in the adsorption of xylene isomers and have less adsorption capacity. The shell particles show high adsorption capacity and moderate selectivity for $\mathrm{p}$-xylene. The preference in adsorption of $\mathrm{p}$-xylene has greatly increased when core-shell particle is used adsorbent. However, the core-shell adsorbent shows intermediate adsorption capacity which perhaps is the combined effect of core and shell since the core has low adsorption capacity. A similar report has been found in literature by Bouizi et al where they studied the adsorption capacity of hydrocarbon molecules using $\beta$-zeolite/silicalite-1 coreshell adsorbents. They found that the core 
has higher adsorption capacity than shell while the core-shell adsorbent shows the intermediate capacity of core and shell [15].

\section{Modeling of Adsorption Column Dynamics}

The breakthrough curves for $\mathrm{p}$-and $\mathrm{o}$ xylene have been discussed in the previous section. Our next goal is to model this curves using the isotherm parameters estimated. Since the concentration of xylenes in the nitrogen stream is rather small, the experiment can be assumed to be a trace system operated under isothermal conditions. Under such conditions, the mass balance of adsorbing component can be written as,

$$
\frac{\partial c_{i}}{\partial t}=\mathcal{D}_{L} \frac{\partial^{2} c_{i}}{\partial z^{2}}-v \frac{\partial c_{i}}{\partial z}-\left(\frac{1-\epsilon}{\epsilon}\right) \frac{\partial q}{\partial t}
$$

and the solid phase mass transfer can be written using the linear driving force approximation,

$$
\frac{\partial q}{\partial t}=k\left(q_{i}^{*}-q_{i}\right)
$$

where $k$ is the LDF coefficient and $q_{i}^{*}$ is the solid phase concentration in equilibrium with the fluid phase concentration.

In this study the binary adsorption isotherm is assumed to follow the competitive Langmuir model, i.e.

$$
q_{i}^{*}=\frac{\mathcal{H}_{i} c_{i}}{1+b_{1} c_{1}+b_{2} c_{2}}
$$

The adsorption of the carrier gas, $\mathrm{N}_{2}$, is neglected. The model described above was solved using finite volume with 100 grid points.

\section{Comparison of Experimental Breakthrough Data and Model Calculations for Single Components}

A dynamic model of fixed bed chromatographic adsorption process was applied to describe the single component experimental breakthrough data. The experimental data and model calculations for concentration profile of $p$-xylene breakthrough experiment are shown in Figure 9. The model parameters obtained from the fitted Langmuir adsorption isotherm are used in the model calculations.

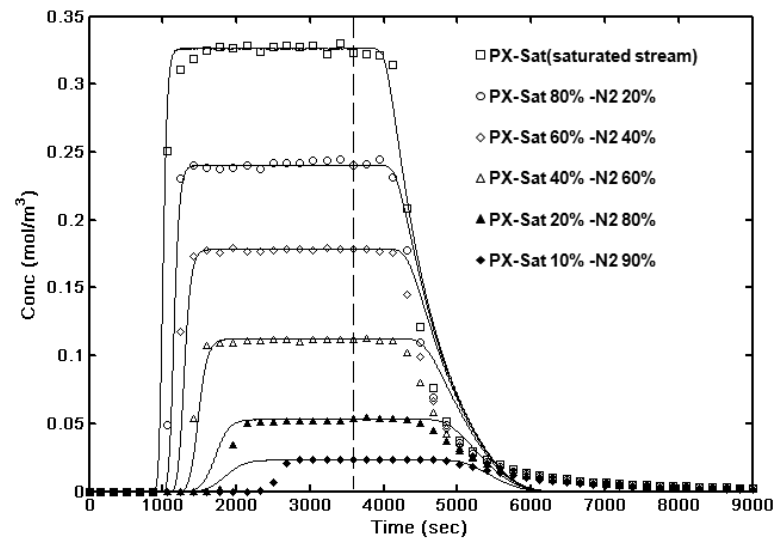

Figure 9: Comparison of Experimental (Symbols) and Model (Lines) Concentration Profiles for Pure p-Xylene with a Constant Isothermal Flow of $10 \mathrm{~mL} / \mathrm{min}$ at $423 \mathrm{~K}$

The solid lines of Figure 9 represent simulated breakthrough profiles while the symbols represent experimental data. The dotted line in Figure 9 represents the time at which the desorption was started. The mass transfer coefficient of $\mathrm{p}$-xylene is adjusted to fit the experimental data and its value was found to be $0.2 \mathrm{~m} / \mathrm{s}$. It can be seen from Figure 9 that the experimental data are well described by the model calculations. While the description of the high concentration runs is very good there are some deviation observed for the low concentration runs. This comes from the fact the high concentration runs have a higher weight for the fitting of the Langmuir isotherms. It is worth noting that calculated profiles have a shorter breakthrough time compared to the experimental one. This suggests that the true value of the Henry's constant and hence the 
selectivity is larger than the estimated values.

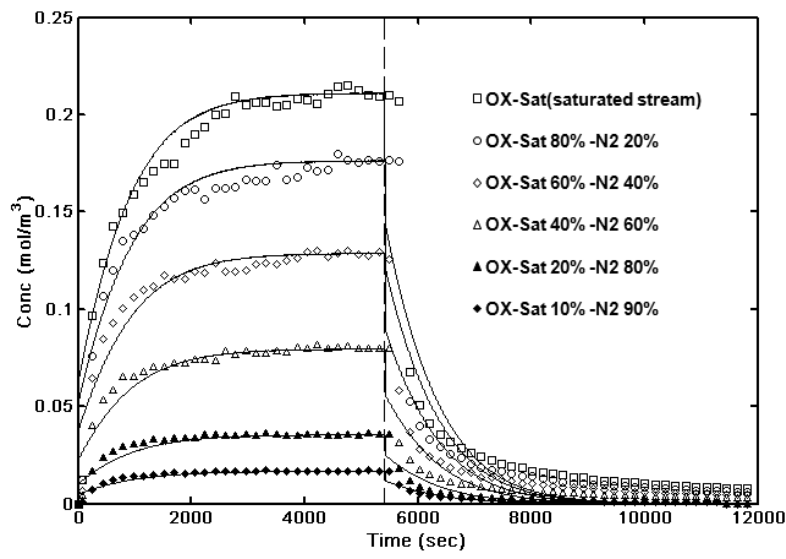

Figure 10: Comparison of experimental (symbols) and model (lines) concentration profiles for pure o-xylene with a constant isothermal flow of $10 \mathrm{~mL} / \mathrm{min}$ at $423 \mathrm{~K}$

Figure 10 shows the model calculations (lines) with experimental breakthrough data (symbols) for the case of pure o-xylene. A good agreement is observed between the experimental breakthrough data and numerical model calculations. A long tail of $\mathrm{o}$-xylene adsorption isotherm is observed for each breakthrough curve. This indicates that $\mathrm{o}$-xylene has high mass transfer resistance to diffuse through shell of the core-shell adsorbent. From the curve fitting, the value of mass transfer coefficient obtained for oxylene is $2 \times 10^{-3} \mathrm{~m} / \mathrm{s}$ while the value adjusted is $0.2 \mathrm{~m} / \mathrm{s}$ for $\mathrm{p}$-xylene.

\section{Breakthrough Curves for Binary Mixtures of Xylene Isomers}

The normalized concentration profiles of binary breakthrough experiments for different set of compositions are shown in Figure 11. Three sets of $(29 / 71,56 / 44$ and 79/21) p/o-xylene mixtures were used to perform the binary adsorption breakthrough experiments. The experimental conditions were as same as that of single component. Two streams of $\mathrm{N}_{2}$ gas were bubbled through the liquid p-xylene and o-xylene and mixed together before the inlet of the adsorption column. The feed composition was varied by adjusting the flow rate of carrier gas.

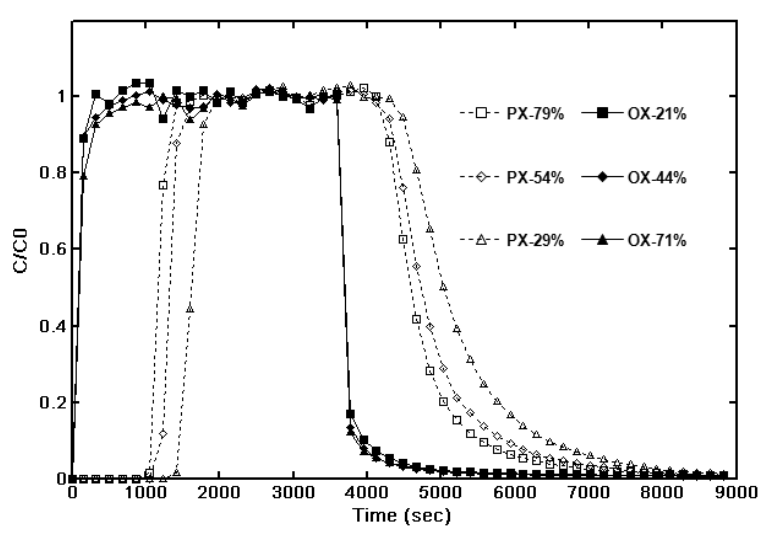

Figure 11: Breakthrough Curves of Binary Mixtures of p/o-Xylene Isomer at $423 \mathrm{~K}$ with Different Feed Concentrations Ratio

In the case of binary mixtures, the significant degree of separation selectivity can be seen from their breakthrough curves. It is worth noting that the response shows constant pattern for o-xylene which indicates linear adsorption isotherm. However, for p-xylene, the adsorption isotherm can be considered favorable based on the indication of breakthrough responses. For both components, the trends are similar to their single component responses. It is worth noting that the classical roll-up effects seen in binary mixtures are absent. This can be explained by the fact that since o-xylene exhibits a linear isotherm, the effect of competitive adsorption is indeed minimal.

\section{Prediction of Experimental Breakthrough Data for Binary Mixture of Xylene Isomers}

From the observation on the breakthrough experiments, we find that there has no significant difference in their dynamic breakthrough behaviors between 
the cases of single component and for binary mixtures. Thus, we attempted to predict the shape of the binary experimental breakthrough curve by applying exactly the same Langmuir and adsorption model parameters that were used in single component model calculations. The predicted model calculations and experimental breakthrough data of binary mixtures are shown in Figure 12. Symbols are experimental data and lines are model calculations. One can see from Figure 12 that the dynamic adsorption model can successfully predict the shape of the breakthrough curve for each of individual component. The model calculations of binary breakthrough again confirm that there has no significant competitive adsorption between p/o-xylene isomers. Hence the separation selectivity of xylene isomer is solely driven by the effect of molecular sieving by the shell.

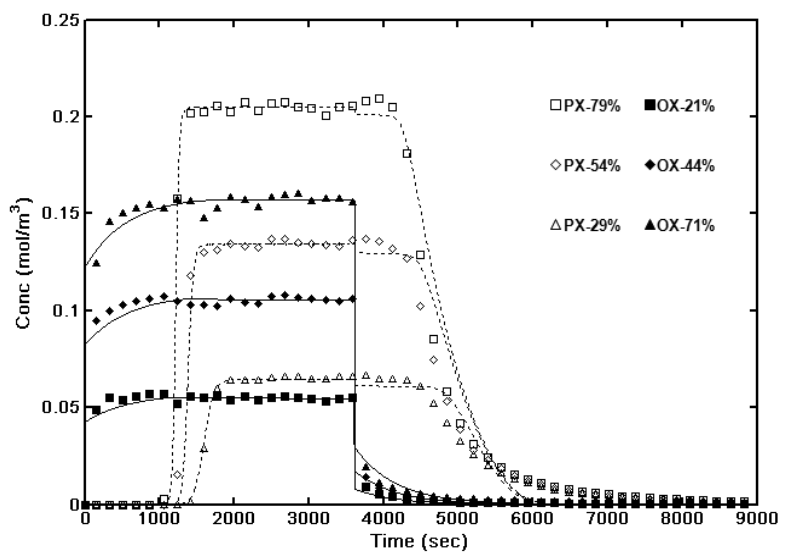

Figure 12: Prediction of Binary Adsorption Breakthrough Curve using Single Component Adsorption Model Parameters, Symbols are Experimental Data and Lines are from Model Prediction

\section{CONCLUSION}

The report has successfully demonstrated a potential application of zeolite core-shell composites as shape selective adsorbents for the gas phase separation of p/o-xylene isomers. The core-shell adsorbents have shown significantly high para-selectivity. The synthesized core-shell particle has a very thin microporous shell and hence offers fast transport rate of molecules. The results obtained from the single and binary breakthrough behaviors confirm that the shell provide sufficient barrier for o-xylene selectively adsorb a significant amount of pxylene from its isomer p/o-mixtures. The effect of mass transfer resistance is much more evident for o-xylene from the single component breakthrough experiments. The LDF coefficient of o-xylene is 100 times lower than that of $\mathrm{p}$-xylene. The paraselectivity obtained from the breakthrough experiment of binary p/o-xylene mixtures is as high as 15. Moreover, the mathematical model applied to represent the adsorption processes has shown a good agreement with the experimental behaviors. The model has successfully predicted the shape of the breakthrough profiles of binary mixtures of xylene isomer by using single component Langmuir isotherm parameters.

\section{REFERENCES}

[1] Ruthven, D. M., Past progress and future challenges in adsorption research. Ind. Eng. Chem. Res. 2000, 39, (7), 2127-2131.

[2] BÃjrcia, P. S.; Silva, J. A. C.; Rodrigues, A. E., Separation by fixed-bed adsorption of hexane isomers in Zeolite BETA pellets. Ind. Eng. Chem. Res. 2006, 45, (12), 4316-4328.

[3] Jolimaitre, E.; Ragil, K.; TayakoutFayolle, M.; Jallut, C., Separation of mono- and dibranched hydrocarbons on silicalite. Aiche Journal 2002, 48, (9), 1927-1937.

[4] Dolye, R. A.; Williams, B. A.; Miller, $J$ T. Para-xyelene selective adsorbent composition and methods. US 2008/0200742 A1, 2008. 
[5] Davis, S.; Y, a. I., CEH Marketing Research report: Zeolites. SRI Consulting 2009.

[6] Tarditi, A. M.; Horowitz, G. I.; Lombardo, E. A., Xylene isomerization in a ZSM-5/SS membrane reactor. Catal. Lett. 2008, 123, (1-2), 7-15.

[7] Lipscomb, G. G., Membrane Separation Technology: Past, Present, and Future. In Innovations in Industrial and Engineering Chemistry, American Chemical Society: 2008; Vol. 1000, pp 281 333.

[8] Caro, J.; Noack, M., Zeolite membranes - Recent developments and progress. Micropor. Mesopor. Mater. 2008, 115, (3), 215-233.

[9] Ferreira, A. F. P.; MittelmeijerHazeleger, M. C.; Bliek, A., Can alkane isomers be separated? Adsorption equilibrium and kinetic data for hexane isomers and their binary mixtures on MFI. Adsorption 2007, 13, (2), 105-114.

[10] Ruthven, D. M.; Reyes, S. C., Adsorptive separation of light olefins from paraffins. Micropor. Mesopor. Mater. 2007, 104, (1-3), 59-66.

[11] Nishiyama, N.; Miyamoto, M.; Egashira, Y.; Ueyama, K., Zeolite membrane on catalyst particles for selective formation of p-xylene in the disproportionation of toluene. Chem. Commun. 2001, (18), 1746-1747.

[12] Khan, E. A.; Rajendran, A.; Lai, Z., Synthesis of $\mathrm{Ni}-\mathrm{SiO}_{2} /$ Silicalite-1 core-shell micromembrane reactors and their reaction/diffusion performance. Ind. Eng. Chem. Res. 2010, 49, (24), 12423-12428.

[13] Casado, C.; Bosque, J.; Navascués, N.; Téllez, C.; Coronas, J., Propane and 1,3,5-triisopropylbenzene single gas adsorption on hollow silicalite-1 spheres. Micropor. Mesopor. Mater. 2009, 120, (1-2), 69-75.

[14] M. Miyamoto, T. K. N. N. Y. E. K. U., Single crystals of ZSM5/Silicalite composites. Adv. Mater. 2005, 17, (16), 1985-1988.

[15] Bouizi, Y.; Diaz, I.; Rouleau, L.; Valtchev, V. P., Core-shell zeolite microcomposites. Adv. Funct. Mater. 2005, 15, (12), 1955-1960.

[16] Santacesaria, E., Separation methods of xylene isomers Chimica \& L Industria 1980, 62, (4), $317-$ 322.

[17] Carra, S.; Santacesaria, E.; Morbidelli, M.; Storti, G.; Gelosa, D., Separation of xylenes on- $Y$ Zeolites .3. Pulse curves and their interpretation. Ind. Eng. Chem. Res. Process Des. Dev. 1982, 21, (3), 451-457.

[18] Santacesaria, E.; Morbidelli, M.; Servida, A.; Storti, G.; Carra, S., Separation of xylenes on-Y Zeolites .2. Breakthrough curves and their interpretatoin Ind. Eng. Chem. Res. Process. Des. Dev. 1982, 21, (3), 446-451.

[19] Santacesaria, E.; Morbidelli, M.; Danise, P.; Mercenari, M.; Carra, S., Separation of xylenes on- $Y$ Zeolites .1. Determination of the adsorption equilibrium parameters, selectivities, and mass-transfer coefficient through finite bath experiments. Ind. Eng. Chem. Res. Process. Des. Dev. 1982, 21, (3), 440-445.

[20] Cottier, V.; Bellat, J.-P.; SimonotGrange, M.-H.; Methivier, A., Adsorption of p-Xylene/m-Xylene Gas Mixtures on BaY and $\mathrm{NaY}$ Zeolites. Coadsorption Equilibria and Selectivities. J. Phy. Chem. B 1997, 101, (24), 4798-4802. 
[21] Bellat, J.-P.; Simonot-Grange, M.H.; Jullian, S., Adsorption of gaseous p-xylene and m-xylene on $\mathrm{NaY}, \mathrm{KY}$, and BaY zeolites: Part 1. Adsorption equilibria of pure xylenes. Zeolites 1995, 15, (2), 124130.

[22] Bellat, J.-P.; Simonot-Grange, M.H., Adsorption of gaseous p-xylene and m-xylene on $\mathrm{NaY}, \mathrm{KY}$, and $\mathrm{BaY}$ zeolites. Part 2: Modeling. Enthalpies and entropies of adsorption. Zeolites 1995, 15, (3), 219-227.

[23] Mellot, C.; Simonot-Grange, M.-H.; Pilverdier, E.; Bellat, J.-P.; Espinat, D., Adsorption of Gaseous p-or mXylene in BaX Zeolite: Correlation between Thermodynamic and Crystallographic Studies. Langmuir 1995, 11, (5), 1726-1730.

[24] Dessau R, M., Selective Sorption Properties of Zeolites. In Adsorption and Ion Exchange with Synthetic Zeolites, AMERICAN CHEMICAL SOCIETY: 1980; Vol. 135, pp 123135.

[25] Morbidelli, M.; Storti, G.; Carra, S., Comparison of adsorption separation processes in the liquid and vapor phase. Application to the xylene isomer mixture. Ind. Eng. Chem. Fund. 1986, 25, (1), 89-95.

[26] Storti, G.; Santacesaria, E.; Morbidelli, M.; Carra, S., Separation of xylenes on-Y Zeolites in the vapor-phase .3. Choice of the suitable desorbent Ind. Eng. Chem. Process Des. Dev. 1985, 24, (1), 8992.

[27] Santacesaria, E.; Gelosa, D.; Danise, P.; Carra, S., Separation of xylenes on-Y Zeolites in the vaporphase .1. Determination of the adsorption equilibrium parameters and of the kinetic regime Ind. Eng.
Chem. Process Des. Dev. 1985, 24, (1), 78-83.

[28] Morbidelli, M.; Santacesaria, E.; Storti, G.; Carra, S., Separation of xylenes on-Y Zeolites in the vaporphase .2. Breakthrough and pulse curves and their interpretation Ind. Eng. Chem. Process Des. Dev. 1985, 24, (1), 83-88.

[29] Khan, E. A.; Hu, E.; Lai, Z. P., Preparation of metal oxide/zeolite core-shell nanostructures. Micropor. Mesopor. Mater. 2009, 118, (1-3), 210-217.

[30] Lai, Z. P.; Bonilla, G.; Diaz, I.; Nery, J. G.; Sujaoti, K.; Amat, M. A.; Kokkoli, $\quad$ E.; $\quad$ Terasaki, O.; Thompson, R. W.; Tsapatsis, M.; Vlachos, D. G., Microstructural optimization of a zeolite membrane for organic vapor separation. Science 2003, 300, (5618), 456-460.

[31] Lai, Z. P.; Tsapatsis, M.; Nicolich, J. R., Siliceous ZSM-5 membranes by secondary growth of b-oriented seed layers. Adv. Funct. Mater. 2004, 14, (7), 716-729.

[32] Lai, Z. P.; Tsapatsis, M., Gas and organic vapor permeation through b-oriented MFI membranes. Ind. Eng. Chem. Res. 2004, 43, (12), 3000-3007.

[33] Snyder, M. A.; Tsapatsis, M., Hierarchical nanomanufacturing: From shaped zeolite nanoparticles to high-performance separation membranes. Angew. Chem., Int. Ed. 2007, 46, (40), 7560-7573.

[34] Zhang, C.; Hong, Z.; Gu, X. H.; Zhong, Z. X.; Jin, W. Q.; Xu, N. P., Silicalite-1 Zeolite Membrane Reactor Packed with HZSM-5 Catalyst for meta-Xylene Isomerization. Ind. Eng. Chem. Res. 2009, 48, (9), 4293-4299. 
[35] Zampieri, A.; Dubbe, A.; Schwieger, W.; Avhale, A.; Moos, R., ZSM-5 zeolite films on Si substrates grown by in situ seeding and secondary crystal growth and application in an electrochemical hydrocarbon gas sensor. Micropor. Mesopor. Mater. 2008, 111, (1-3), 530-535.

[36] Nishiyama, N.; Ichioka, K.; Miyamoto, M.; Egashira, Y.; Ueyama, K.; Gora, L.; Zhu, W.; Kapteijn, F.; Moulijn, J. A., Silicalite-1 coating on $\mathrm{Pt} / \mathrm{TiO} \mathrm{O}_{2}$ particles by a two-step hydrothermal synthesis. Micropor. Mesopor. Mater. 2005, 83, (1-3), 244-250.

[37] Yan, T. Y., Separation of p-xylene and ethylbenzene from $C 8$ aromatics using medium-pore zeolites. Ind. Eng. Chem. Res. 1989, 28, (5), 572576.

[38] Yan, T. Y., Effects of moisture in separation of $\mathrm{C} 8$ aromatics using medium-pore zeolites. Ind. Eng. Chem. Res. 1988, 27, (9), 16651668.

[39] Alaerts, L.; Maes, M.; Giebeler, L.; Jacobs, P. A.; Martens, J. A.; Denayer, J. F. M.; Kirschhock, C. E. A.; De Vos, D. E., Selective Adsorption and Separation of orthoSubstituted Alkylaromatics with the Microporous Aluminum Terephthalate MIL-53. J. Am. Chem. Soc. 2008, 130, (43), 14170-14178.

[40] Ruthven, D. M.; Goddard, M., Sorption and diffusion of $C 8$ aromatic hydrocarbons in faujasite type zeolites. I. Equilibrium isotherms and separation factors. Zeolites 1986, 6, (4), 275-282. 